\title{
The polyhomeotic gene of Drosophila encodes a chromatin protein that shares polytene chromosome-binding sites with Polycomb
}

\author{
Marco DeCamillis, Niansheng Cheng, Denise Pierre, and Hugh W. Brock \\ Department of Zoology, University of British Columbia, Vancouver, British Columbia, Canada V6T 1 Z4
}

The Polycomb group (PcG) genes in Drosophila melanogaster are required for maintenance of correct spatial expression of homeotic genes, and their products are thought to form either a regulatory network or act as a multimeric complex. Recently, it has been suggested that because of homology between Polycomb (Pc) and $\mathrm{Su}$ (var)205, PcG genes encode chromatin proteins required for the maintenance of a determined state in chromatin. The polyhomeotic $(p h)$ gene is a member of the PcG of genes. We present DNA sequence of a ph cDNA, which encodes a 169-kD protein with a single putative zinc finger, a serine/threonine-rich region, and has glutamine repeats, suggesting that $p h$ is a DNA-binding protein. Polyclonal antisera directed against $p h$ protein bind to $\sim 80$ sites on polytene chromosomes. Most of these sites appear to be the same as those recognized by antibodies to $P c$ protein. $p h$ protein binds to insertion sites of constructs containing DNA from the bithoraxoid $(b x d)$ region of the Bithorax complex, showing that ph binding to chromatin is DNA dependent. The same $b x d$ constructs are recognized by $P c$ protein, strongly supporting the hypothesis that $p h$ and $P c$ interact directly.

[Key Words: Drosophila; polyhomeotic gene; Polycomb group; chromatin protein]

Received October 18, 1991; revised version accepted December 4, 1991

Genetic and molecular analyses have shown that spatial regulation of homeotic genes is required for correct determination of segmental identity in Drosophila (Lewis 1978; Akam 1987; Ingham 1988). The homeotic genes of Drosophila are arranged in two complexes: the Antennapedia complex (ANT-C), which contains genes that specify head and thoracic segments (Kaufman et al. 1980; Scott et al. 1983); and the Bithorax complex (BX-C), which specifies thoracic and abdominal segments (Lewis 1978; Bender et al. 1983; Karch et al. 1985). Paro (1990) has pointed out that the early-acting segmentation genes and maternal genes that control homeotic expression pattern (for review, see Ingham 1988) are transiently expressed and that other control mechanims must take over to maintain specific expression or repression of the homeotic genes. Genetic analysis has shown that the Polycomb group (PcG) of genes is required to repress homeotic gene expression (Struhl 1981; Ingham 1984).

PcG mutants exhibit homeotic transformations in embryos and adults similar to gain-of-function mutations in genes of the ANT-C and BX-C. After careful phenotypic analysis of Polycomb (PC) mutants (Lewis 1978), Denell and Frederick (1983) suggested that $P_{c}$ mutations upset the transmission of determined states. Molecular studies have suggested that PcG genes are not needed for correct initiation of spatially regulated homeotic gene expres- sion but, rather, for maintenance of spatial regulation once it has been established (Struhl and Akam 1985; Wedeen et al. 1986; McKeon and Brock 1991). Pc protein binds to polytene chromosome sites that contain the ANT-C and BX-C. Moreover, Pc protein binds to insertion sites of constructs containing regulatory DNA from Antennapedia $(A n t p)$ and Ultrabithorax $(U b x)$ shown to be genetically sensitive to PcG mutant backgrounds in transformed lines (Zink and Paro 1989; Zink et al. 1991; J. Simon, A. Chiang, and W. Bender, unpubl.). These data show that $P c$ binding is DNA dependent and support a direct role for $P C$ in the regulation of homeotic genes.

Many of the PcG genes are pleiotropic, because mutations in various loci can exhibit segmentation defects (Ingham 1984; Breen and Duncan 1986), central nervous sytem defects (Smouse et al. 1988), cuticle defects (Dura et al. 1987), or were isolated because they interact with zeste (Wu et al. 1989; Jones and Gelbart 1990; Brunk et al. 1991). Zink and Paro (1989) have shown that PC protein binds to $\sim 60$ sites on polytene chromosomes, a number higher than expected for a specific regulator of the homeotic genes. These data suggest that PcG genes are involved in a common process that can affect different target genes.

Paro and Hogness (1991) showed that $P c$ has significant homology to $\mathrm{HPl}$, a heterochromatic protein that is 
the product of the Su(var)205 locus (Eissenberg et al. 1990). Modifiers of position-effect variegation (PEV) are thought to be chromatin proteins (for review, see Henikoff 1990). We have shown that several PcG genes act as modifiers of PEV (D. Sinclair, N. Clegg, T. Grigliatti, and $\mathrm{H}$. Brock, in prep.). These results are consistent with the idea that PcG genes are somehow required for establishment or maintenance of chromatin structure. A number of investigators have suggested that $P c$ regulates homeotic genes by imprinting, or promoting regional heterochromatinization, which would render cis-regulatory elements inaccessible to transcription factors, rather than by acting as transcription factors themselves (Alberts and Sternglanz 1990; Gaunt and Singh 1990; Gyurkovics et al. 1990; Paro 1990; Reuter et al. 1990). Detailed analysis of each PcG product will provide a basis for dissecting this process.

Two hypotheses have been advanced to explain why PcG genes have overlapping functions. Double and triple mutations of Polycomblike, Additional sex combs, Posterior sex combs, and Sex combs on midleg show marked enhancement of homeotic transformations, similar to strong alleles of Pc or extra sex combs (esc), compared with the relatively weak phenotypes associated with any single mutation. These interactions may reflect a regulatory hierarchy within the PcG (Jürgens 1985). Support for this idea comes from the studies of Zink and Paro (1989), who showed that Pc protein recognizes the locations of seven PcG genes on polytene chromosomes. An alternative explanation for these synergistic effects has been proposed by Locke et al. (1988), who suggested that PcG gene products may be single components of a multimeric complex. Such a complex would follow laws of mass-action kinetics for macromolecular assembly and thus be sensitive to dosage changes in PcG products. As described below, indirect evidence from analysis of polyhomeotic $(p h)$ supports the mass-action model. However, the two models may not be mutually exclusive, as some PcG proteins could form a complex that participates in a regulatory hierarchy.

In this work we extend the molecular analysis of $p h$. $p h$ is genetically complex (Dura et al. 1987), and ph mutants exhibit pleiotropic phenotypes (Dura et al. 1985, 1987; Smouse et al. 1988; Santamaria et al. 1989; Smouse and Perrimon 1990|. Two genetic lesions are required on the same chromosome to create null or strong hypomorophic alleles, whereas weak hypomorphs result from any single-lesion alleles. These results show dosage sensitivity of the $p h$ locus and support the mass-action model of PcG function. The ph locus is arranged as a tandem duplication contained within $25 \mathrm{~kb}$ of genomic DNA (Dura et al. 1987). Long stretches of $80-100 \%$ sequence identity exist between duplicated regions that correspond closely to long open reading frames (ORFs) (Deatrick et al. 1991). Analysis of genomic long ORFs suggests that a ph protein encoded by either repeat contains a single putative zinc finger, in addition to other domains commonly found in DNA-binding proteins. Together, these data suggest that $p h$ contains two transcription units that encode functionally similar proteins that may interact with DNA.

Here, we characterize three cDNAs that map to the proximal repeat of the $p h$ locus and show that the protein it encodes contains a possible zinc finger motif, a serine/threonine-rich region, and glutamine repeats, consistent with a role for $p h$ in DNA and protein interactions. Antisera directed to $p h$ protein shows localization of the ph product to $\sim 80$ sites on polytene chromosomes. We find that extensive overlap exists between the binding sites of $p h$ and $P c$ on polytene chromosomes. Finally, we show that the $p h$ protein binds in vivo to the insertion site of a construct containing a $14.5-\mathrm{kb}$ fragment of bithoraxoid $(b x d)$ regulatory DNA that is genetically sensitive to ph as well as other PcG mutant backgrounds. These results are discussed in light of current models of PcG function.

\section{Results \\ Isolation and sequencing of proximal ph cDNAs}

As described in the introductory section, the $p h$ locus is genetically complex. Northern analysis has identified two major embryonic transcripts that are encoded by $p h$ (Dura et al. 1987), which map to either the proximal or distal repeat (N. Randsholt and S. Freeman, unpubl.). Together, these data suggest that ph encodes two homologous transcription units, one located in the proximal and one in the distal repeat. To confirm that the genomic long ORFs encode $p h$ transcripts, we have cloned and sequenced cDNAs from the proximal repeat. We screened an imaginal disc library and purified 28 clones (see Materials and methods). Three overlapping clones, $c 4.3, c 4-6$, and $c 4-11$, that mapped to most of the proximal ph repeat were subcloned into plasmid vectors and sequenced (Fig. 1). The structure of $p h$ will be character-
Figure 1. Cloning of ph proximal cDNA. Three overlapping clones were isolated from an imaginal disc library constructed in $\lambda$ gt 10 obtained from G. Rubin. The sizes and relative positions of each clone, C4.3, C4-6, C4-11, are indicated under the restriction map of the proximal transcription unit (X) XhoI; (S) SalI; (H) HindIII; (B) BamHI).

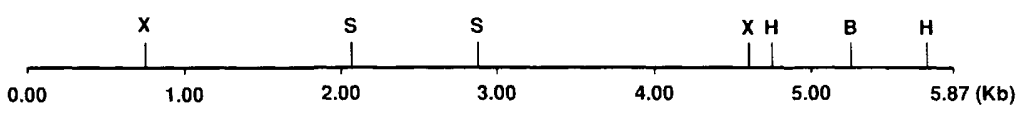

C4.3 $(4.17 \mathrm{~Kb})$

C4-6 (5.70 Kb)

C4-11 (5.68 Kb) 
ized elsewhere (N. Randsholt, N. Cheng, S. Freeman, and $\mathrm{H}$. Brock, in prep.). Complete sequence of these clones was obtained using a combination of directed deletions (Henikoff 1984) and synthesis of oligonucleotide primers (Fig. 2A).

These data indicate that the overlapping clones together comprise a nearly full-length proximal cDNA of $5.8 \mathrm{~kb}$ that is missing some of the $5^{\prime}$-untranslated region but includes the complete coding sequence of the structural protein (Fig. 2A). There are two candidate ATG codons for the translation start, but we believe that the $5^{\prime}$-most codon is the probable start because there is an in-frame stop codon just upstream and reasonable agreement with the consensus start sequence (Kozak 1984). Some minor differences in the cDNA sequence compared with the genomic sequence (Deatrick et al. 1991) are detailed in the legend to Figure 2. Some occur at third positions in codons and presumably reflect DNA polymorphisms between strains, and others are revisions of the sequence. Most notably, we have detected a novel intron that splices out $63 \mathrm{bp}$ of ORF sequence that is perfectly conserved between the repeats. In addition, we have identified $\sim 200$ amino acids at the amino terminus that were not identified from the genomic sequence because this identification depended on sequence conservation between the proximal and distal repeats (Deatrick et al. 1991).

Conceptual translation of the proximal cDNA is presented in Figure 2A, and the location of protein motifs is summarized in Figure 2B. This cDNA encodes a 169-kD protein that contains a single potential $\mathrm{C} 4$ class zinc finger domain (Evans and Hollenberg 1988). The putative zinc finger has two pairs of cysteines separated by 2 and 3 amino acids, respectively, which are, in turn, separated by 14 residues, 7 of which are basic. These basic residues could react with DNA.

Several other putative functional domains have been identified. A region is present upstream of the zinc finger from amino acid 1233 to 1290 , in which 39 of 58 amino acids are either serine or threonine. Four glutamine-rich regions are found in the amino-terminal $60 \%$ of the protein. A prediction of protein structure identified a potential $\alpha$-helix-forming region on the carboxy-terminal end of the $p h$ protein (Chou and Fasman 1978). This putative helix contains partial similarity to the second helix of the helix-loop-helix ( $\mathrm{HLH})$ consensus sequence found in several classes of HLH proteins (Villares and Cabrera 19871 .

A homology search for related proteins using the FASTP program (Pearson and Lipman 1988) with the PIR data bank found no significant matches to proteins sequenced previously. It is notable that $p h$ protein has no "chromobox" or other sequence homology with Pc (Paro and Hogness 1991).

ph encodes a 169-kD chromatin protein that binds to specific sites on polytene chromosomes

To investigate the $p h$ protein further, we raised polyclonal antisera directed against $p h$ products. A proximal
cDNA fragment (Fig. 2A), composed of sequences completely conserved between the proximal and distal repeats (Deatrick et al. 1991), was cloned into a $\beta$-galactosidase expression vector. Antibodies to this peptide will react with all known ph products $(\mathrm{N}$. Cheng, unpubl.). Western blots of protein extracts from bacterial expression cultures containing the expression vector with and without the ph cDNA demonstrate that the antiserum only recognizes epitopes from the ph portion of the fusion protein (Fig. 3). The antiserum does not react with any other proteins in Western blots of proteins from embryos homozygous for a deletion that includes $p h$ (Fig. 3, lane 6) and thus is specific for $p h$. The native $p h$ protein was detected on a Western blot from salivary gland extracts prepared from third-instar larvae (Fig. 3, lane 5). A prominent band corresponding to a molecular mass of $\sim 170 \mathrm{kD}$ is evident, which is consistent with the molecular mass predicted from conceptual translation of the proximal cDNA analysis above. Other bands may be minor $p h$ products (N. Randsholt, unpubl.) or degradation products.

The $P c$ protein has been found in salivary glands, and it binds to $\sim 60$ sites on polytene chromosomes (Zink and Paro 1989). The $p h$ antiserum was used to stain polytene chromosomes and shows site-specific binding (Fig. 4A). Analysis of the binding sites shows that $p h$ recognizes $\sim 80$ sites distributed throughout the genome ( $\mathrm{Ta}$ ble 1). We compared the best nuclei from many independent polytene preparations and included only those sites that were present in the majority of nuclei.

Comparison of Table 1 and the Pc-binding sites published by Zink and Paro (1989) shows a large degree of overlap. To compare $p h$ - and $P c$-binding sites more directly, we simultaneously determined the distribution of $P c$ antibody (kindly provided by $\mathrm{R}$. Paro) and ph antibody, using chromosomes from different nuclei that had been prepared identically. In general, the strong $P c$ - and $p h$-binding sites appear to be indistinguishable /Fig. 4B,Cl. We have detected additional PC-binding sites not listed in Zink and Paro (1989), and these are indicated in brackets in Table 1. In addition, we have made some revisions of the cytology where the $p h$ and $P C$ sites appear indistinguishable, and these changes are indicated in parentheses in Table 1. Overall, at least 71 of $78 \mathrm{ph}$ binding sites appear to overlap with $P c$-binding sites.

\section{ph protein binds to constructs containing bxd DNA}

It is not clear from the above results whether $p h$ binding to polytene chromosomes is DNA sequence dependent or whether $p h$ associates with some chromatin regions independent of the underlying sequence. Simon et al. (1990) have constructed transformation vectors containing $l a c Z$ reporter genes under the regulation of sequences from the $U b x$ region of the $\mathrm{BX}-\mathrm{C}$. One of these, containing $14.5 \mathrm{~kb}$ of DNA from the $b x d$ region at coordinates -18.5 to -4.0 on the BX-C walk, directs reporter gene expression that respects appropriate parasegmental boundaries in wild-type embryos. However, in PcG mutant embryos, including $\mathrm{Pc}^{-}$and $\mathrm{ph}^{-}$embryos, ectopic 


\section{DeCamillis et al.}

A

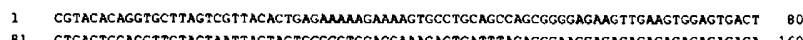
B1 GTGAGTGGAGGTTGTACTAATTACTACTGCCCGTGCACGAAACACTCATTTACACGCAAGCACACACACACACACACACA 160 161 CACACGAGACACTGGGACTTTTGTCGGATTTTCGTATTCGTTTTGTGTACTTT?GTTGTCTTGCGTTCCACGTTACATAC 240 241 ATATG:ATATGTTTGGTGTTGCCTGTTGTATGT:TATATTTATTGCCTTCACACATGTGCGTGTTTGITAATGTACAhIA 320 321 TAATACGGCAM:AGCAAMAMGAGAAGAMACTGAGGACTAAAMAGAMAGCGCCATGCCGACTATMMCAAAAACAMCCACG 400 401 AGTGTCTCCGCCGCCGCCGAAAACAAAhACAAAACGCCTAAGCCGACGCATGCATACETATAATTTATTRTAAATATTGT 480

481 TTTTATTTTGMTMUTGGATCGTCGTGCATTGAMGTTTATGCAMAMAGAGGGGACACAGMAGCGATACGACCACACCC 560 1 M D R R A L K F M O K R A D T E S D T T T P 22 561 GTGAGCACCNCAGCATCCCAGGGGATTTCAGCATCAGGGATTCTAGCAGGAGGCACTCTTCCCTTGAAGGACAACTCGAA 640 23 V S T T T A S O G I I S A 5 A A I L A A G G I L L P L L K 641 CATCGGCGaGaAGCCCCTCCACCATAACTACAACCACAATAACAaCAaCAGCTCCCAGCACTCACACTCGCACCAGCAGC 720 50 I R E K P L L H H N Y Y H H N N N N S S O K S H S H Q Q 721 AGCAACAACAGCAGGTGGGTGGCAAGCAGCTCGAGCGGCCACTAAAGTGCCTGGAAACGCTCGCCCAGAAGGCGGGAATC 800

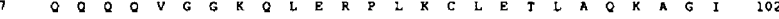
801 ACCTTCGAGGAGMATACGATGTGGCCAGCCCCCCGCATCCCGGCATTGCCCAGCAGCAGGCGACTTCAGGAACAGGCCC OBO 103 T F D E K Y X D V A A S P F H P G I A O O O O A I S G T 891 AGCAACGGGATCAGGCTCAGTCACCCCCACAAGCCATCGGCACGGAACTCCGCCCACGGGCCGCAGGCAAACCCACACCC 960 130 A I G S G S 961 CAAGCACTCCGMACAGGCCCAGTGCTCCCAGCACACCCAACACTAACTGCAACTCAATTCCCCGCCACACCAGCCTCACG 1040

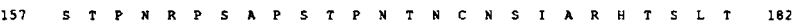
1041 CTGGAGAAGGCGCAGMATCCCGGCCAGCAGGTGGCCGCGACCACCAGGSTGCCACTGCAGATATCCCCTGAGCAGCTGCA 1120 183 \& E K A O N P G O O Y A A T T T Y P L O I S P E O L O 209 1121 GCAGTTCTATGCGAGCAATCCCTACGCCATTCAGGTGAaGCANGAGTTTCCCACGCACACGACCAGTGGCAGTGGAACTG 1200

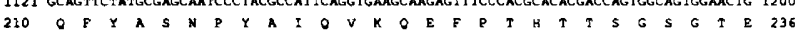
1201 MACTAAGCATGCAACCMCATTATGGMGTTCAGCAGCAGTTGCAGCTGCAGCAGCAGCTGTCGGAMGCCMACGGTGGA 1280 237 L K H A T N I M E V O O O L Q L O O O L L S E A N G G 262 1281 GGAGCAGCCTCGGCCGGAGCCGGAGGAGCAGCTAGTCCGGCCAACTCGCAGCAAAGCCAGCAACAGCAGCACTCCACAGC 1360 263 G A A S A G A G G A A S P A N S $Q$ O S 1361 CATCAGCACCATGTCGCCGATGCAATTGGCAGCGGCCACTGGAGGAGTTGGCGGGGATTGGACACAGGGAMGGACGGTGC 1490 290 I S T M S P M O L A A A T G G V G G O T O G R T V O 326 1441 AGCTAATGCAACCCTCCACCAGTTTCCTGTATCCCCAAATGATTGTGTCGGGAAATCTGTTGCATCCAGGAGGCCTCGGT 1520 317 L M Q P S I I S E L Y Y P O M I V V S G N I I I H P G G L G 312 1521 CAGCAGCCAATCCAGGTGATCACCGCCGGCAMGCCATTCCAAGGCAACGGCCCCCAGATGCTTACCACCACGACTCAMM 1600 343 Q Q P I O V I T A G K P F Q G N G P O M L T T T T O N 369 1601 CGCCMAGCAMATGATCGGTGGCCAAGCGGGATTCGCTGGCGGAMATTACGCGACCTGCATTCCCACAAACCACAATCAAT 1680 370 A K O M I G G O A G F A G G N Y A T C I P I N K N 1691 CGCCCCAGACGGTGCTCTTCTCACCGATGAACGTCATTTCGCCACAGCAGCAACAGAACCTGCTGCMATCAATGGCCGCT 1760 397 P O I V V L F S P M N V I I S P

1761 GCAGCTCAGCAGCAGCANTTCACCCMACAGCAGCAACAGTTJMACCAGCAGCAACAACAGCAGCTTACTCAGCAGCMACA 1840

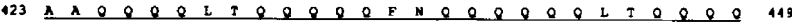
1841 GCAGTTGACAGCTGCTCTGGCCAAGGTGGGAGTGGATGCGCAGGGCMAGCTGGCCCAGMUATGGT:CAGAAAGTGACTA 1920 450 \& $L$ I A A L A K K V G V D A O G K I A A O K V V O O K V V T T 476 1921 CCACCAGTAGCGCGGTCCAGGCGGCGACGGGTCCTGGATCTACTGGGTCMACACAGACCCAGCAGGTGCAGCAGGTTCAG 2000 477 T S S A V O A A T G P G S T G S T

2002 CAACAGCAGCAGCAGACCACCCAAACCACTCAGCAGTGCGTGCAGGTTTEACAGTCGACTTTGCCAGTCGGTGTGGGTGG 2080 503 \& Q Q Q Q I T Q I T Q Q C V Q V S Q S I L P V G V G G 529 20B1 ACAGTCTGTTCAGACTGCCCAACTTCTGMCGCTGGCCAAGCGCAACMARTGCAGATTCCCTGGTTCTTACAGMTGCTG 2160 530 O S V Q T A O L L N A G Q A Q O M Q I P N F L O N A A 556 2161 CAGGACTGCAGCCGTTTGGGCCAMACCAGATCATCCTGCGMMCCAGCCAGACGGAACCCMAGGCNTGTTCATTCMACAG 2240 $557 G L$ L $P$ F G P N O I I L R N O P D G I O G M F I O O 582 2241 CAACCGGCGACGCAGACTTTGCAGACCCAGCAMAACCAGATTATTCMATGCAACGTGACGCAGACGCCCACTAAGECACG 2320 583 O P A T O T L O T O O N Q I I O C N V T Q T P T K A R 609 2321 CACTCMCTGGATGCACTTGCTCCCMAGCAGCMCAGCAGCNGCAGCAGGTTGCCACTACEAACENGACGCAGCAGCAGC 2400

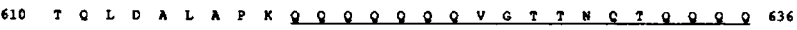
2401 MACTAGCGGTGGCTACTGCCCAGTTGCAGCMCAGCAGCAGCAMCTCACTGCAGCAGCTCTGCAGCGACCAGGAGCCCCT 2480 637 L A V A T A Q L Q Q Q Q Q Q S I I A A A I O A P G A P 662 24B1 GTCATGCCCCACMATGMACTCAAGTGCGTCCGGCCAGTICCGTATCCACACAGACTGCCCAGAACCAGAGCCTGCTGAA 2560 663 Y M P H N G T O V R P A S S Y S T Q T A O K O S I L K 689 2561 GGCCMAMTGGGCMACMCCAGCAICCGGTGCGCCCCGCTTTAGCCACATTGMAMCOGMATCGGTĆMAGTCGCAGGAC 2640 590 A X M R N K O P V R P A L T T X T I G V A G O 116 2641 MMATMAGGTAGTAGGCCACCTGACCACCGTCCAGCAGCAGCMCAGGCGACGMTCTCCAGCAGGTGGTTMTGCGGCT 2720 717 N K V V G H L T T V O O O O OATNLEO V V NA A 142 2721 GGCMCMUATGGTTGTGATGAGCACMCGGGCACTCCGATCACCCTGCAGMTGGACAGACCCTTCATGCMGCCACTGC 2800 743 G N X M V V A S T T G T P I T L O N G O T L H A A T A 769 2001 GGCAGGAGTCGACANGCAGCAMCAGCAGCTACANCTGTTTCAGMACMGCMUTCCTGCAACANCAACAMTGTTGCMAC $28 B 0$

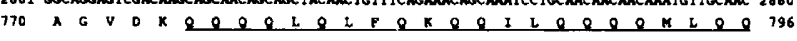
2881 AGCAGATTGCTGCCATTCAMATGCAGCAGCAGCNGGCGCTGTTCAGGCCCACCMCNACAGCAGCANCACGTCTCTCAG 2960

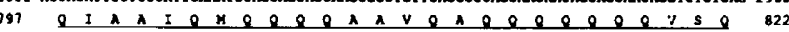

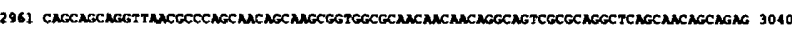

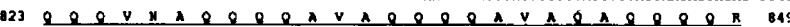

3041 GCAGCAACAGCAGCAAGTTECCCAAGCCCAGGCGCACCATCAACAGGCTCTCGCGAATGCCACTCAGCAAATCCTTCAGG 3120

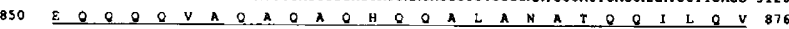
3121 TGGCGCCAAATCAATTCATCACGTCCCACCAGCAACAGCAGCAGCAGCAACTTCACAACCAACTGATACAGCAGCAGCTA 3200

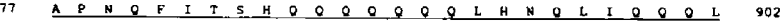
3201 CAGCAACAGGCGCAGGCACAAGTTCAAGCCCAAGTGCAGGCTCAAGCGCAACAGCAACAACAGCAGCGAGAGCAGCAGCA 3280

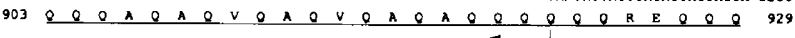
3281 GAATATTATCCAGCAGATTGTGGTGCAACAGTCTGGAGCGACTTCTCAACAGACTTCCCAGCAGCAACAGCACCACCAMT 3360

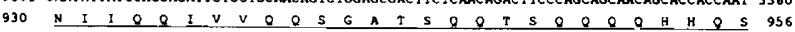
3361 CEGGGCAACTACAGCTAAGTAGCGTGCCCTTCTCAGTTTCTTCGTCAACGACGCCAGCCGGAATAGCTACCTCTAGTGCT 3440

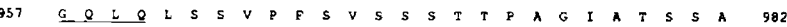
3441 CTGCAGGCAGCCCTCTCCGCCTCTGGCGCCATCT:TCAGACAGCTMGCCGGGTACTTGCAGTTCCTCCTCCCCCACAAG 3520 983 L \& A A I S A S G A I F O T A K F G T C S S S S S P T S 1009 3521 CAGTGTGGTCACAATTACCAACCAGAGCAGCACTCCTTTGGTCACCAGCAGTACGGTGGCCAGTATACAGCAGGCTCAGA 3600 1010 S V V T I T N O S S T P L V T S S T V A S I O OA T 1036 3601 CGCAATCTGCTCAGGTCCACCAACATCAGCAGCTAATCAGCGCCACAATTGCCGGAGGGACTCAACMCAGCCACAGGGA 3680 1037 Q S A O V H O HOOE I S A T I A G G O O O O G 1062 3681 ССGCCATCACTTACACCCACCACAAATCCAATTTTGGCCATGACCTCGATGATGMATGCTACAGTGGGTCACCTTTCCAC 3760 1063 P P S L T P T T N P I L A M T S M M N A T V G H L S T 1089 3761 TGCTCCGCCTGTMACTGTTTCTGTGACAAGCACCGCTGTTACTTCGTCGCCGGGTCAGCTGGTTCTCTTAAGCACGGCTA 3840 1090 A P F V T V S V T S T A V T S S F G O L V L L S T A S 1116 3841 GTAGCGGTGGAGGAGGTAGCATACCAGCCACGCCCACCAMAGAGACACCTTCGAMGGGCCCACCGCAMCCCTGGTGCCC 3920

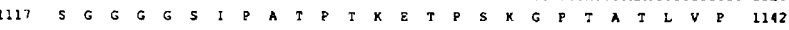
3921 ATTGGTTCGCCCAAGACTCCTGTATCAGGMAGGACACCTGCACTACCCCLAMATCATCTACTCCTGCCACTGTCAGCGC 1000 1143 I G S P K T T P V S G K O O T C T T P P K S S T P P A T V S S A 1169 4001 ATCCGTAGAGGCCAGTAGTTCCACAGGCGMAGCCCTGTCCAATGGAGATGCCTCAGATAGGTCTTCCACGCTGTCAMAGG 4080 1270 S VE A S S S T GEA L S N G D A S O R S S T L S K G 1196 4081 GCGCTACCACTCCCACCAGCAAGCAAAGCAATGCAGCAGTGCAGCCACCGAGTAGCACCACTCCCAMCAGTGTCAGTGGG 1160 1197 A T I P T S K O S N A A V O P P S S T T P N S V S G 1222 1161 AMAGMAAGCCGMAGCTGGCAACCTGCGGCAGTTTAMCGTCCGCMACATCMCTTCMACCACGACMACGATCACCMTGG 1240 $1223 K E E E P K$ L A T C G S L T S A T S T S T T T T I T N G 1249 4241 GATTGGAGTAGCCAGAACGACAGCCAGCAGGGCTGTCTCAACCGCTAGCACAMCCACTACCAGTTCTGGCACCTTTATCA 4320

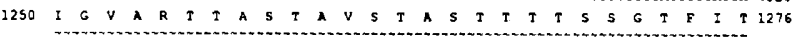
4321 CAAGTTGCACCAGCACMACCACAACCACCACGTCGAGTATCAGTMATGGATCGMGGATCTCCCCMMGGCGATGATTMAG 4400

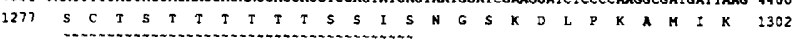
1401 CCGAACGTCTTAACTCACGTCATCGATGGCTTCATCATCCAGGAGGCCMACGAGCCATTTCCCGTCACCAGACAGCGATA 4480 1303 P N V L I I H V V I D $G$ F I 4481 TGCAGACMAAGACGTCAGCGATGAGCCGCCMAMGMMMAGGCMACCATGCAGGAGGACATCAMGCTMGGGGMATAGCAT 1560 1330 A D K D V S D E P P K K K A T M O E D I K L S G IA S 1356 1561 CAGCTCCAGGCTCGGATATGGTTGCTTGCGAGCAGTGTGGMAAGATGGAGCACMAMGCAMAGCTGAMACGGAAGCGCTAC 1640 357 A P G S D M V A C I O C G K X I X \& X L X X X X 1382 4641 TGTTCGCCAGGATGCTCGAGGCAGGCMUAGMCGGCATCGGTGGAGTTGGATCAGGAGAGACGNACGGCCTGGGGACAGG 4720 1383 C P C C S R O A X N G I G G V G S G E T N G L G T G 1109 1721 TEGTATAGTTGGGGTGGACGCCATGGCATTGGTGGACAGGCTGGATGMAGCCATGGCTGAGGAGMAGATGCAGACAGAGG 1800 2410 G I V G V D A X A L V O R I O E A A A E E K A Q T E A 1436 4801 CCACCCCAMAGCTTTCAGAATCGTTTCCTATTTTGGGAGCCTCMACAGMGTACCTCCMATGTCACTGCCAGTCCAMGCG 4880 4037 T F K L S E S F F I L G A S T R V P P M S L P V Q A 1462 4981 GCGATTTCTGCGCCCTCGCCTCTTGCANGCCTCTAGGATCGCCATTGTCAGTTGCACTTCCMACTCTTGCACCACTGTC 4960 1463 A I S A P S P L A $M$ P L 4962 TGTAGTCACTTCTGGCGCGGCGCCCMAGTCTTCGGMGGTGAMTGGMAGAGATCGTCCGCCMATCAGCAGCTGGAGTGTGG 5040 1490 V V T S G A A P KS S E V NG T D R P P I S S N S V D 1516 5041 ACGATGTCAGCAACTTCATTCGAGAACTGCCTGGTTGTCAGGACTACGTGGACGACTTTATACAGCAGGAGATCGACGGC 5120 1517 D V S N F I R E L P G C O D Y V D D F I O O E I D S121 CMAGCGCTTCTGTTGCTCAMGGAGAAGCATTTGGTGAMCGCTATGGGCATGMGCTGGGTCCAGCTCTTMAMTTGTGEC 5200 1543 O A L L L L K E K H L V N A H G M K L G P A L K I V A 1569 5201 CMMGGTGGAGTCCATTMAGGAGGTCCCGCCACCTGGCGAGGCCMMGGATCCAGGAGCGCNGTAGGGCAGCTAGNGCACGA S280 1570 K V E S I K E V F P P G E A K D P G A O . 5281 MAGCCGMUMAGATGATCTCCTMACCGAGCAGTGGACCTGGTTCMCCMGTCTGTCGTGCCAGGTGATTCTGATTCM 5360 S361 TCGGGCAGGCGAMUGGACGCGMATCCATTTGCAMAMTATTAT TAGCATCAGGCCATCAGGTCTTAMACCCATTGACTTT 5140 5411 GTACATACTCCCAAGATCACTRATMGCATATTCATTTATMMTTMMCTMCAGTCMCAGTCMMUСGMTGGMTT 5520

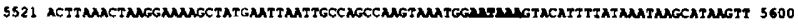
5601 TAZAGTCTAMGTAGCATATTAATMACTCCCMCG TCATGGATAGTTTGTACMNGTATTTTAATCTTAGGMTCMATGTA 5680

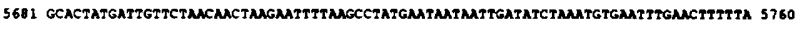

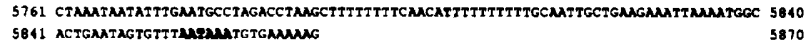

Figure 2. (See facing page for legend.)

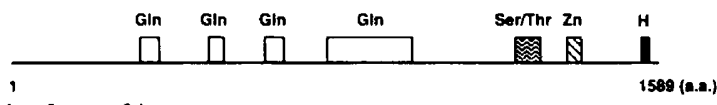


expression of the reporter is observed, suggesting that this $b x d$ DNA is a potential target for PcG genes (J. Simon, A. Chiang, and W. Bender, pers. comm.). Furthermore, experiments with antibodies to $P c$ show that $P c$ protein binds at the insertion sites of this $b x d$ construct in two transformed lines, 85J-23 and 85-39, confirming that this DNA is a target for PC binding (A. Chiang, W. Bender, and R. Paro, pers. comm.). As shown in Figure $5 \mathrm{~B}$, a new site of $p h$ binding is detected in the transformed line 85-39 compared with the wild-type pattern shown in Figure 5A, and the new site corresponds to the insertion site of the bxd DNA (Fig. $5 \mathrm{C}$ ). We have also shown that ph binds to the insertion site of $b x d$ DNA in line $85 \mathrm{~J}-23$ (results not shown). These results demonstrate that $p h$ binding to polytene chromosomes is DNA sequence dependent.

\section{Discussion}

\section{Features of the ph protein}

The putative ph zinc finger has not been shown to bind zinc and is not homologous to individual zinc fingers of the steroid receptor class (S. Henikoff, pers. comm.), even though it contains 4 cysteine residues (Evans and Hollenberg 1988; Vallee et al. 1991). It resembles a similar unique cysteine array found in domain 3 of the adenovirus E1A protein (Moran and Mathews 1987), in which the 4 cysteines coordinate to a single zinc atom as a requirement for trans-activation (Gulp et al. 1988). E1A does not bind DNA directly (Ferguson et al. 1985) and may interact with proteins already bound to promotor complexes (Lillie and Green 1989). It is possible that the putative $p h$ zinc finger has a similar role for protein interaction in chromatin.

\section{ph targets on polytene chromosomes}

Consistent with deductions about $p h$ function derived from cDNA sequence analysis, we show that $p h$ is a chromatin protein that is present at $\sim 80$ sites on polytene chromosomes. Strong sites always stain, but there is more variability in weak sites, probably because of minor differences in the squashing technique, flatness of the preparation, or because the chromosomes are in different puffing stages. All of these differences might limit

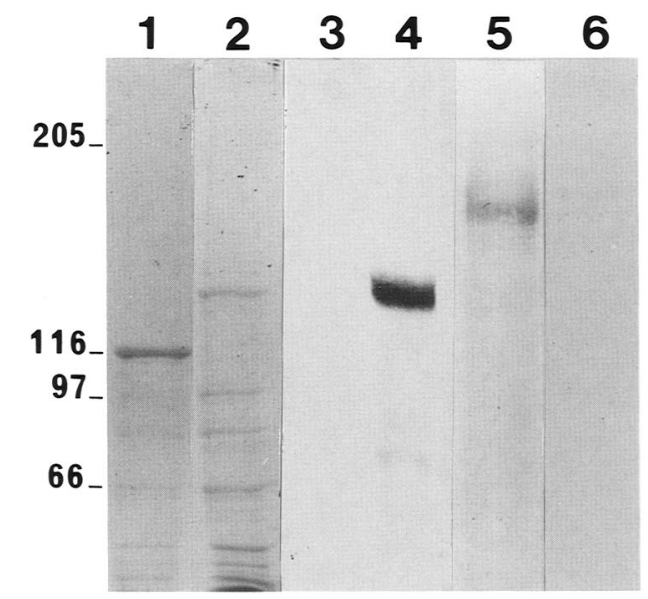

Figure 3. Antibodies against the ph protein. Antisera were raised against a $l a c Z-p h$ fusion. Crude bacterial lysates were prepared from expression clones containing lac $Z$ (lanes 1,3 ) or lacZ-ph fusions (lanes 2,4) and separated on 7\% SDS-acrylamide gels. Gels were either stained with Coomassie blue (lanes 1,2) or electroblotted onto nitrocellulose and reacted with $p h$ antiserum, and binding was detected with secondary antibodies labeled with horseradish peroxidase (Vector Labs) (lanes 3,4). (Lane 5) Salivary gland lysates from 100 third-instar larvae were separated on 7\% SDS-acrylamide gels and electroblotted to nitrocellulose, and the filters were reacted with ph antisera, as described above. (Lane 6) A Western blot of proteins prepared from 50 embryos homozygous for a deletion, $D f(1) J A 52$, that uncovers $p h$. ph null embryos were identified by their phenotype, prepared as described in Materials and methods, and proteins were separated electorophoretically and transferred to nitrocellulose. The filter was reacted with ph antiserum at a concentration $10 \times$ higher than that shown in lane 5 . No crossreacting antigens were detected, showing that the antibody is specific to $p h . P c$ antibodies reacted with similar samples did detect $P_{c}$, showing that the integrity of the proteins from the $p h$ null embryos was maintained. Molecular mass of protein standards is shown at left (in $\mathrm{kD}$ ).

the accessibility of the $p h$ antibody to the $p h$ protein and increase the likelihood that weak sites may be missed. Therefore, the list presented is a minimum estimate of ph-binding sites.

Comparison of $P c$ - and ph-binding sites on different preparation has limitations. Some stem from the vari-

Figure 2. The nucleotide sequence of $p h$ proximal cDNA. The near full-length $p h$ proximal cDNA sequence, including the complete coding strand and predicted amino acid sequence, is shown in $A$. Two polyadenylation sequences at the $3^{\prime}$ end of the transcript are shown in boldface type. Six ATG codons occur 5' to the ORF start but are followed by in-frame termination codons. $p h$ antisera were raised against fusion proteins constructed using the cDNA fragment shown from amino acids 753 to 922 (arrows). The location of putative motifs is indicated. Blocks of glutamine-rich sequences are underscored with solid lines. A single domain in which 39 of 58 amino acids are hydroxylated serine or threonine is underscored with tildes (1233-1290). A region containing partial homology to the HLH motif is underscored with a broken line (1549-1562). A potential C4 zinc finger lies between amino acids 1365 and 1387 (boldface type). The relative positions of these features are summarized in $B$. The following differences between the genomic sequence reported by Deatrick et al. (1991) and the cDNA sequence reported here were noted (cDNA sequence is listed second): (nucleotide 4) G $\rightarrow$ A (change in noncoding region); (nucleotide 110) $\mathrm{T} \rightarrow \mathrm{C}$ (change in noncoding region); (nucleotide 1266) insertion of AGC (results in insertion of Gln); (nucleotide 2720) G $\rightarrow$ T (no change); (nucleotides 4738-4739) CA $\rightarrow$ AC (changes Ala to Asp); (nucleotide 5364) $A \rightarrow G$ (change in noncoding region). 
Figure 4. Immunohistochemical localization of $p h$ protein on polytene chromosomes. (A) Polytene chromosome preparations from wild-type animals were reacted with $p h$ antisera and detected using horseradish peroxidase-conjugated secondary antibody (Vector Labs). Only the autosomes are shown here. In $B$ and $C$, proximal is on the left and distal is on the right. $(B)$ Detail of $p h$ binding to the distal part of chromsome $2 \mathrm{R}$ from a wild-type strain, taken under bright-field illumination. Ordered from distal to proximal, the cytological positions of ph binding are $60 \mathrm{E}, 59 \mathrm{~F}, 59 \mathrm{C}$, $58 \mathrm{~F}, 58 \mathrm{D}, 57 \mathrm{~B}, 57 \mathrm{~A}$, and $56 \mathrm{C}$. Note that a unique $p h$ site occurs at $58 \mathrm{~F}$ (arrowhead). $(C)$ Detail of $P c$ binding to distal $2 \mathrm{R}$, taken under phase-contrast illumination. Note that most $P C$ sites appear to be equivalent to $p h$ sites but that the $58 \mathrm{~F}$ site is absent.

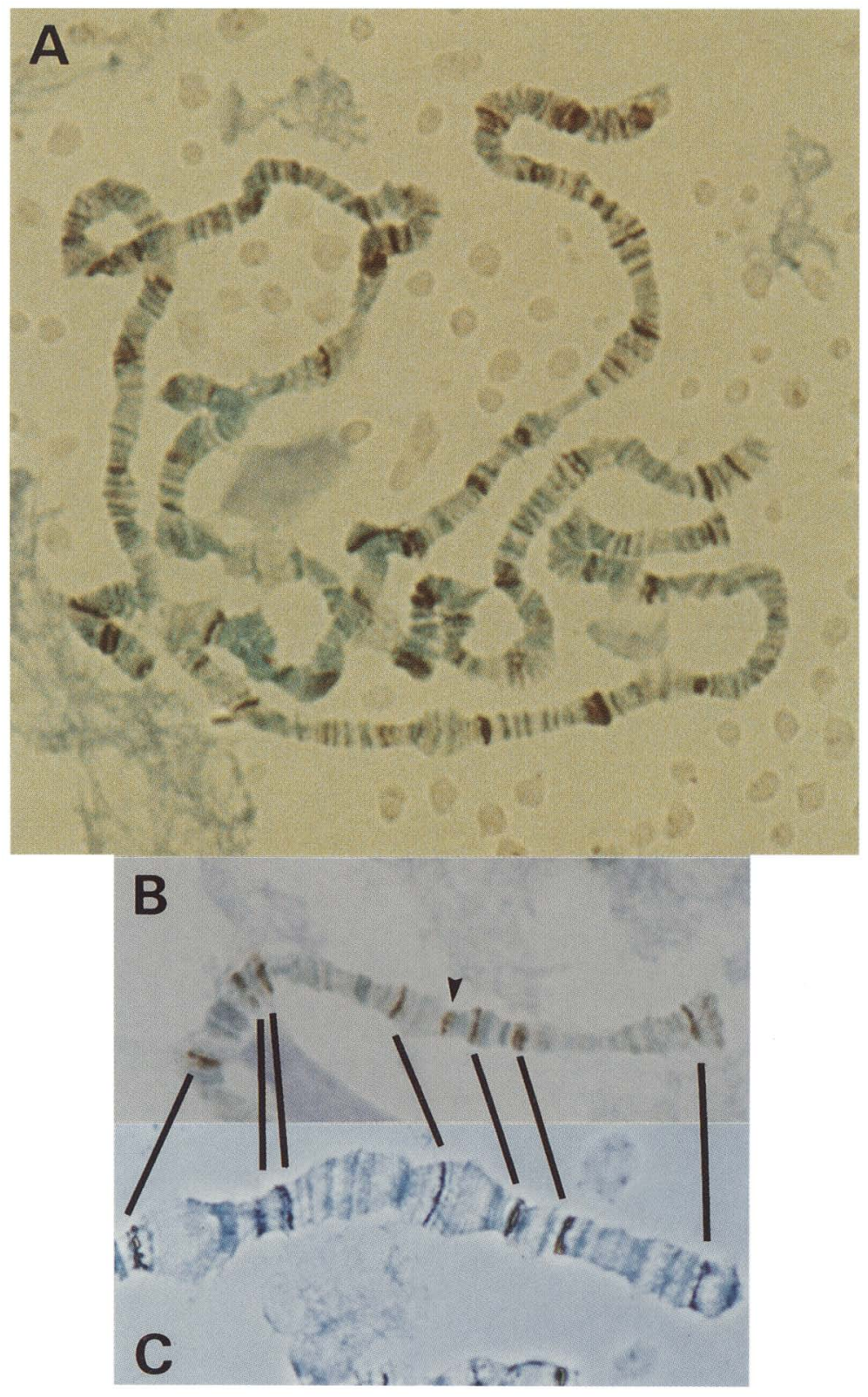

view of the differences between $p h$ and $P c$ phenotypes (Denell and Frederick 1983; Dura et al. 1987), it is not surprising that there appear to be differences between $p h$ - and Pc-binding sites. Overall, these data strongly support the idea that PcG genes have overlapping, but not identical, functions.

The segmentation genes even-skipped and engrailed, and homeotic genes are ectopically expressed in $\mathrm{ph} \mathrm{mu-}$ tants, suggesting that $p h$ is a repressor of these genes (Dura and Ingham 1988; Smouse et al. 1988; McKeon and Brock 1991). As expected, $p h$-binding sites have been identified in the regions known to contain these loci: $46 \mathrm{C}, 48 \mathrm{~A}, 84 \mathrm{AB}$, and $89 \mathrm{E}$ in the case of even-skipped, engrailed, ANT-C, and BX-C, respectively (Lindsley and Zimm 1985). Positive identification of any gene as a tar- 
Table 1. Comparison of $\mathrm{ph}$ and Pc protein-binding sites on polytene chromosomes

\begin{tabular}{|c|c|c|c|c|c|c|c|}
\hline \multicolumn{2}{|c|}{ X chromosome } & \multicolumn{2}{|c|}{ Chromosome 2} & \multicolumn{2}{|c|}{ Chromosome 3} & \multicolumn{2}{|c|}{ Chromosome 4} \\
\hline$p h$ & $P_{c}$ & $p h$ & $P c$ & $p h$ & $P_{c}$ & $p h$ & $P c$ \\
\hline- & 1B & $21 \mathrm{~A}$ & & $61 \mathrm{~A}$ & {$[61 \mathrm{~A}]$} & $102 \mathrm{AC}$ & \\
\hline $2 \mathrm{D}$ & & $22 \mathrm{~A}$ & & $61 \mathrm{C}$ & & - & $102 D$ \\
\hline $4 \mathrm{C}$ & & $22 B$ & $(22 \mathrm{C})$ & $61 \mathrm{~F}$ & {$[61 F]$} & & \\
\hline $5 \mathrm{~A}$ & & $24 \mathrm{~A}$ & & $62 \mathrm{~F}$ & (63A) & & \\
\hline $5 \mathrm{D}$ & & $25 \mathrm{EF}$ & & $65 \mathrm{D}$ & & & \\
\hline $7 \mathrm{~B}$ & & $26 \mathrm{~F}$ & & $66 \mathrm{E}$ & - & & \\
\hline $8 \mathrm{~A}$ & & $28 \mathrm{~A}$ & [28A] & $67 \mathrm{D}$ & & & \\
\hline $8 \mathrm{~B}$ & & $29 \mathrm{E}$ & & $67 \mathrm{E}$ & $|67 F|$ & & \\
\hline $9 \mathrm{~A}$ & & $32 \mathrm{EF}$ & & 67EF & {$[67 \mathrm{EF}]$} & & \\
\hline $12 \mathrm{D}$ & & $33 F$ & & $69 \mathrm{C}$ & & & \\
\hline $14 \mathrm{~B}$ & (14A) & $35 \mathrm{AB}$ & - & $69 \mathrm{D}$ & & & \\
\hline $16 \mathrm{D}$ & {$[16 \mathrm{D}]$} & $35 \mathrm{C}$ & & $70 \mathrm{C}$ & $(70 A)$ & & \\
\hline $17 \mathrm{~A}$ & - & $36 \mathrm{AC}$ & $(36 \mathrm{~B})$ & $70 \mathrm{DE}$ & [70DE] & & \\
\hline & & $37 \mathrm{~A}$ & {$[37 \mathrm{~A}]$} & $76 \mathrm{~B}$ & - & & \\
\hline & & $37 \mathrm{~B}$ & {$[37 \mathrm{~B}]$} & $78 \mathrm{E}-79 \mathrm{~B}$ & - & & \\
\hline & & $38 \mathrm{~F}$ & & $82 \mathrm{E}$ & & & \\
\hline & & $41 \mathrm{CD}$ & & $84 \mathrm{D}$ & & & \\
\hline & & $43 C$ & & $84 \mathrm{~F}$ & & & \\
\hline & & $44 \mathrm{~A}$ & & $86 \mathrm{C}$ & & & \\
\hline & & $45 \mathrm{C}$ & & $88 \mathrm{~A}$ & & & \\
\hline & & $46 \mathrm{CD}$ & & $89 \mathrm{~B}$ & & & \\
\hline & & $48 \mathrm{~A}$ & & $89 \mathrm{C}$ & & & \\
\hline & & $49 \mathrm{EF}$ & & $89 \mathrm{E}$ & & & \\
\hline & & $51 \mathrm{~A}$ & & $90 \mathrm{E}$ & & & \\
\hline & & $56 \mathrm{C}$ & & $93 \mathrm{E}$ & & & \\
\hline & & $57 \mathrm{~A}$ & {$[57 \mathrm{~A}]$} & $94 \mathrm{D}$ & - & & \\
\hline & & $57 \mathrm{~B}$ & {$[57 \mathrm{~B}]$} & $94 \mathrm{E}$ & {$[94 \mathrm{E}]$} & & \\
\hline & & $58 \mathrm{D}$ & $\{58 \mathrm{D}\}$ & $96 \mathrm{C}$ & (96A) & & \\
\hline & & $58 \mathrm{~F}$ & - & $98 \mathrm{~B}$ & $(98 \mathrm{~A})$ & & \\
\hline & & $59 \mathrm{C}$ & $|59 \mathrm{C}|$ & $99 \mathrm{AB}$ & {$[99 \mathrm{AB}]$} & & \\
\hline & & $59 \mathrm{~F}$ & & $99 \mathrm{~F}$ & & & \\
\hline & & $60 \mathrm{E}$ & & $100 D$ & {$[100 D]$} & & \\
\hline & & & & $100 \mathrm{E}$ & {$[100 \mathrm{E}]$} & & \\
\hline
\end{tabular}

For clarity, only changes to the $P C$-binding sites identified by Zink and Paro $(1989)$ are listed here. If the $P_{C}$ column is blank, the observed $p h$-binding sites are the same as those given by Zink and Paro (1989). Pc sites that we have detected, but that are not listed in Zink and Paro (1989), are enclosed in brackets. Where we believe the ph site is equivalent to the $P_{C}$ site, but disagree with the cytology of Zinc and Paro (1989), the original $P_{C}$ cytology is given in parenthesis. Sites not detected with $p h$ or $P c$ antibody are indicated with a dash.

get of ph binding will require transformation studies such as we have carried out with the bxd DNA fragment. A systematic survey of putative ph-binding sites should eventually allow identification of most $p h$ targets, which would greatly increase our understanding of the pleiotropic phenotypes of the PcG.

Uncertainties in the cytology of ph- and Pc-binding sites, and in the mapping of putative target genes, means that any list of potential targets of ph binding is speculative. Nevertheless, in view of the similarities between Pc and Su(var)205 (Paro and Hogness 1991) and our observation that the majority of PcG genes, but not ph itself, also modify PEV (D. Sinclair, N. Glegg, T. Grigliatti, and $\mathrm{H}$. Brock, in prep.), it is interesting that ph appears to bind to four regions containing known locations of modifiers of PEV, including E(var) 26A (25EF); Su(var)3-4 (84D), Su(var)3-14 (86CD), and Su(var)3-11 (94D-95A) (Lindsley and Zimm 1990). It is also interesting that $p h$ appears to bind to many of the locations containing other PcG genes. If this speculation is correct, ph may have an activating function for some targets, because most PcG proteins are required continuously in development.

It might be expected that most of the ph-binding sites identified on polytene chromosomes represent sites of inactive genes, consistent with chromatin-imprinting models (Gaunt and Singh 1990; Paro 1990), and the observation that $p h$ is a negative regulator of homeotic and segmentation genes (Dura and Ingham 1988; Smouse et al. 1988). Genes active in the salivary gland that are regulated by ph might not be identified.

The observation that $p h$ binds to a $b x d$ construct ectopically located on polytene chromosomes demonstrates that $p h$ recognition of the target site is DNA sequence dependent. This result makes it unlikely that $p h$ protein recognizes general chromatin configurations found in limited positions in the genome independently of the DNA. However, this result does not ascertain 


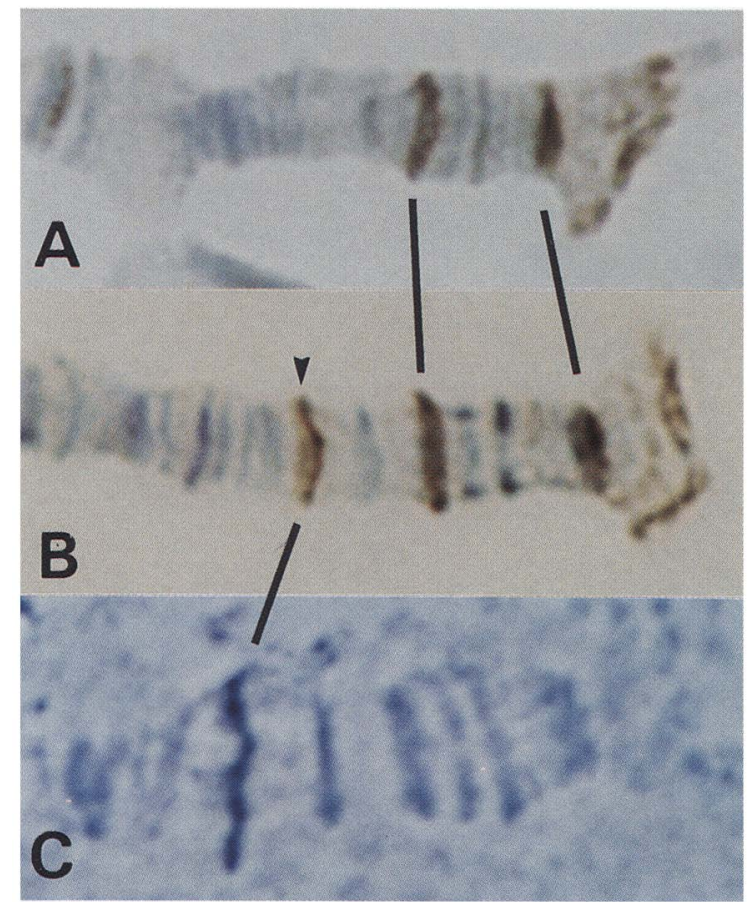

Figure 5. Localization of $p h$ to $b x d$ regulatory sequences in vivo on chromosome $3 \mathrm{~L}$. All chromosomes are oriented with proximal on the left and distal on the right. $(A) p h$-binding sites on wild-type chromosomes. $(B)$ ph binding to the transformed line $85-39$, containing $14.5 \mathrm{~kb}$ of DNA from the $b x d$ enhancer (Simon et al. 1990). A new site of $p h$ binding appears relative to wild type (arrowhead). $(C)$ In situ hybridization of Carnegie 20 DNA, containing the rosy locus to polytene chromosomes of strain 85-89. Note that the site of insertion of the bxd transformant corresponds to the new ph-binding site shown in $B$.

whether $p h$ interacts directly with DNA or indirectly via proteins that recognize DNA themselves. It will be interesting to determine whether $p h$ protein can bind DNA directly. It will also be interesting to determine the number and location of $p h$-binding sites within a region containing a gene.

Locke et al. (1988) proposed that the PcG proteins may form a multimeric complex. The overlap of $p h$ - and $P c$ binding sites supports this hypothesis. The observation that both $p h$ and $P c$ recognize constructions containing the 14.5-kb bxd fragment provides further evidence for this idea. It will now be necessary to perform similar experiments on successively smaller pieces of target DNA to locate the target more precisely and to determine whether $p h$ and $P C$ sites are separable. However, confirmation of this proposal awaits the molecular demonstration of interactions between PcG proteins. Such studies are under way in our laboratory.

Our results are consistent with the hypothesis that PcG proteins are chromatin proteins that associate to regulate the establishment or maintenance of chromatin structure. High-resolution studies of the location and distribution of PcG-binding sites on homeotic genes provide a promising opportunity to increase our understanding of chromatin regulation. These studies may lead to wider understanding of the importance of chromatin structure to gene regulation.

\section{Materials and methods}

\section{General methods}

Routine library screening, subcloning, and DNA sequencing procedures are described in Sambrook et al. (1989).

\section{cDNA isolation and sequencing}

A cDNA library constructed from imaginal disc mRNA lobtained from Dr. G. Rubin, University of California, Berkeleyl was screened for $p h$ cDNAs using the 4.0-kb Sall fragment from the coordinates 127.5 to 131.5 of the distal repeat as a probe (Dura et al. 1987). Twenty-eight cDNA clones were isolated and rescreened with a proximal-specific probe. Five proximal-specific cDNAs were identified and subcloned into Bluescript or pUC 18 vectors. A combination of directed deletions (Henikoff 1984) and synthesis of oligonucleotide primers was used to sequence three proximal cDNAs and to confirm intron-exon splice iunctions, using T7 polymerase (Pharmacia) and the dideoxynucleotide method of Sanger et al. (1977).

\section{Preparation of antisera}

Fusion proteins were prepared using a bacterial expression system. A 500-bp proximal cDNA fragment (see Fig. 2A) was cloned into pUR 290 (Rüther and Müller-Hill 1983). This vector, called pRE5, was transformed into bacteria and induced to express, and the fusion protein was isolated according to Rio et al. (1986) with minor modifications. Proteins were dialyzed against PBS after urea extraction, cleared by centrifugation, and used immediately for injection or to make affinity columns. Fusion protein $(500 \mu \mathrm{g}-2 \mathrm{mg})$ was used for initial and boost injections into New Zealand white rabbits. Blood was collected after two boost injections, and serum was tested for $p h$-binding activity. To make $p h$-specific antiserum, immunoglobins were precipitated from serum in $50 \%$ ammonium sulfate, resuspended in PBS, dialyzed, and passed successively over three affinity columns containing whole bacterial protein lysates, purified $\beta$-galactosidase, and purified fusion protein, respectively. $p h$-specific antibodies were eluted from the last column and used for Western blots and immunohistochemistry.

\section{Western blot analysis}

Bacterial proteins were prepared from expression cultures of BMH71-18 (Rüther and Müller-Hill 1983) containing pRE5 or pUR 290. Two-milliliter cultures were induced, and bacteria were pelleted by centrifugation, resuspended in protein sample buffer, and separated electrophoretically on a 7\% SDS-polyacrylamide gel (Laemmli 1970). Salivary glands were dissected from 100 third-instar larvae and boiled in $200 \mu$ l of protein sample buffer, and the proteins were separated on SDS-acrylamide gels as described above. Proteins were then electroblotted onto nitrocellulose, processed using an appropriate dilution of primary antibody, and detected with secondary antibody coupled to horseradish peroxidase (Jackson Labs).

To prepare $p h$ null embryos, $D f(1) J A 52 / F M 7$ females were allowed to lay for 3-hr intervals. The embryos were aged for 12 $\mathrm{hr}$ and dechorionated in $50 \%$ bleach. Hemizygous Df(1)/A52/Y 
embryos were recognized by the absence of head involution at stage 14. About 50 embryos were boiled in $100 \mu \mathrm{l}$ of sample buffer and analyzed as described above.

\section{Immunohistochemistry and in situ hybridization}

Staining of salivary glands and polytene chromosomes with $p h$ antibody was performed according to Zink and Paro (1989). Chromosomes were photographed with Fuji ASA 100 slide film under bright-field illumination, although some favorable preparations were photographed under phase-contrast illumination. In situ hybridization was carried out with biotinylated Carnegie 20 DNA as a probe.

\section{Acknowledgments}

We thank R. Paro for the gift of $P c$ antibody, for communicating unpublished data, and for stimulating discussions. We are grateful to W. Bender, A. Chiang, and J. Simon for providing transformed lines, communicating unpublished data, and for helpful cooperation. We thank Anne Taylor-Smith for excellent technical assistance and for help with the computer analysis. This work was supported by a grant from the National Science and Engineering Research Council to H.W.B.

The publication costs of this article were defrayed in part by payment of page charges. This article must therefore be hereby marked "advertisement" in accordance with 18 USC section 1734 solely to indicate this fact.

\section{Note added in proof}

Sequence data described in this paper have been submitted to EMBL/GenBank data libraries.

\section{References}

Akam, M.E. 1987. The molecular basis for metameric pattern in the Drosophila embryo. Development 101: 1-22.

Alberts, B. and R. Sternglanz. 1990. Chromatin contract to silence. Nature 344: 193-194.

Bender, W., M. Akam, F. Karch, P.A. Beachy, M. Peifer, P Spierer, E.B. Lewis, and D.S. Hogness. 1983. Molecular genetics of the bithorax complex in Drosophila melanogaster. Science 221: 23-29.

Breen, T.R. and I.M. Duncan. 1986. Maternal expression of genes that regulate the bithorax complex of Drosophila melanogaster. Dev. Biol. 118: 442-456.

Brunk, B.P., E.C. Martin, and P.N. Adler. 1991. Molecular genetics of the Posterior sex combs/Suppressor 2 of zeste region of Drosophila: Aberrant expression of the suppressor 2 of zeste gene results in abnormal bristle development. Genetics 128: 119-132.

Chou, P. and G. Fasman. 1978. Prediction of the secondary structure of proteins from their amino acid sequences. $A d v$. Enzymol. 47: 145-147.

Culp, J.S., L.C. Webster, D.J. Friedman, C.L. Smith, W.-J. Huang, W.Y.-H. Felicia, M. Rosenberg, and R.P. Ricciardi. 1988. The 289-amino acid Ela protein of adenovirus binds zinc in a region that is important for trans-activation. Proc. Natl. Acad. Sci. 85: 6450-6454.

Deatrick, J., M. Daly, N.B. Randsholt, and H.W. Brock. 1991. The complex genetic locus polyhomeotic in Drosophila melanogaster potentially encodes two homologous zinc-finger proteins. Gene 105: 185-195.

Denell, R.E. and R.D. Fredrick. 1983. Homeosis in Drosophila:
A description of the Polycomb lethal syndrome. Dev. Biol. 97: 34-47.

Dura, J.-M. and P. Ingham. 1988. Tissue- and stage-specific control of homeotic and segmentation gene expression in Drosophila embryos by the polyhomeotic gene. Development 103: $733-741$

Dura, J.-M., H.W. Brock, and P. Santamaria. 1985. Polyhomeotic: A gene in Drosophila melanogaster required for correct expression of segment identity. Mol. Gen. Genet. 198: $220-231$.

Dura, J.-M., N. Randsholt, J. Deatrick, I. Erk, P. Santamaria, J.D. Freeman, S.F. Freeman, D. Weddell, and H.W. Brock. 1987. A complex genetic locus, polyhomeotic, is required for segmental specification and epidermal development in $\mathrm{D}$. melanogaster. Cell 51: 829-839.

Eissenberg, J.C., T.C. James, D.M. Foster-Hartnett, T. Hartnett, V. Ngan, and S.C.R. Elgin. 1990. Mutation in a heterochromatin-specific chromosomal protein is associated with suppression of position-effect variegation in Drosophila melanogaster. Proc. Natl. Acad. Sci. 87: 9923-9927.

Evans, R.M. and S.M. Hollenberg. 1988. Zinc fingers: Gilt by association. Cell 52: 1-3.

Ferguson, B., B. Krippl, O. Andrisani, N. Jones, H. Westphal, and M. Rosenberg. 1985. Ela 13s and 12s mRNA products made in Escherichia coli both function as nucleus-localized transcription activators but do not directly bind DNA. Mol. Cell. Biol. 5: 2653-2661.

Gaunt, S.J. and P.B. Singh. 1990. Homeogene expression patterns and chromosomal imprinting. Trends Genet. 6: 208212

Gyurkovics, H., J. Gausz, J. Kummer, and F. Karch. 1990. A new homeotic mutation in the Drosophila bithorax complex removes a boundary separating two domains of regulation. EMBO I. 9: 2579-2585.

Henikoff, S. 1984. Unidirectional digestion with Exonuclease III creates targeted breakpoints for DNA sequencing. Gene 28: 351-359.

1990. Position-effect variegation after 60 years. Trends Genet. 6: 422-426.

Ingham, P. 1984. A gene that regulates the bithorax complex differentially in larval and adult cells of Drosophila. Cell 37: 815-823.

1988. The molecular genetics of embryonic pattern formation in Drosophila melanogaster. Nature 355: 25-34.

Jones, R.S. and W.M. Gelbart. 1990. Genetic analysis of the Enhancer of zeste locus and its role in gene regulation in Drosophila melanogaster. Genetics 126: 185-199.

Jürgens, G. 1985. A group of genes controlling the spatial expression of the bithorax complex in Drosophila. Nature 316: 153-155.

Karch, F., B. Weiffenbach, M. Peifer, W. Bender, I. Duncan, S. Celniker, M. Crosby, and E.B. Lewis. 1985. The abdominal region of the bithorax complex. Cell 43: 81-96.

Kaufman, T.C., R. Lewis, and B. Wakimoto. 1980. Cytogenetic analysis of chromosome 3 in Drosophila melanogaster: The homeotic gene complex in polytene chromosome interval 84A-B. Genetics 94: 115-133.

Kozak, M. 1984. Compilation and analysis of sequences upstream from the translational start site in eukaryotic mRNAs. Nucleic Acids Res. 12: 857-872.

Laemmli, U.K. 1970. Cleavage of structural proteins during the assembly of head of bacteriophage T4. Nature 227: 680-685.

Lewis, E.B. 1978. A gene complex controlling segmentation in Drosophila. Nature 276: $565-570$.

Lillie, J.W. and M.R. Green. 1989. Transcription activation by the adenovirus Ela protein. Nature 338: $39-44$ 
DeCamillis et al.

Lindsley, D.L. and G. Zimm. 1985. The genome of Drosophila melanogaster part 1: Genes A-K. Drosophila Inf. Serv. 64: 1227.

- Zimm. 1990. The genome of Drosophila melanogaster part 4: Genes L-Z. Drosophila Inf. Serv. 68: 1-382.

Locke, J., M.A. Kotarski, and K.D. Tartoff. 1988. Dosage dependent modifiers of position-effect variegation in Drosophila and a mass action model that explains their effect. Genetics 120: $181-198$.

McKeon, J. and H.W. Brock. 1991. Interactions of the Polycomb group of genes with homeotic loci of Drosophila. Wilhelm Roux's Arch. Dev. Biol. 199: 387-396.

Moran, E. and M.B. Mathews. 1987. Multiple functional domains in the adenovirus Ela gene. Cell 48: 177-178.

Paro, R. 1990 . Imprinting a determined state into the chromatin of Drosophila. Trends Genet. 6: 416-421.

Paro, R. and D.S. Hogness. 1991. The Polycomb protein shares a homologous domain with a heterochromatin-associated protein in Drosophila. Proc. Natl. Acad. Sci. 88: 263-267.

Pearson, W.R. and D.J. Lipman. 1988. Improved tools for biological sequence comparison. Proc. Natl. Acad. Sci. 85: 2444-2448.

Reuter, G., M. Giarre, J. Farah, J. Gausz, A. Spierer, and P. Spierer. 1990. Dependence of position-effect variegation in Drosophila on dose of a gene encoding an unusual zinc-finger protein. Nature 344: 219-223.

Rio, D.C., F.A. Laski, and G.M. Rubin. 1986. Identification and immunochemical analysis of biologically active Drosophila P element transposase. Cell 44: 21-32.

Rüther, U. and B. Müller-Hill. 1983. Easy identification of cDNA clones. EMBO I. 2: 1791-1794.

Sambrook, J., E.F. Fritsch, and T. Maniatis. 1989. Molecular cloning: A laboratory manual, 2nd ed. Cold Spring Harbor Laboratory Press, Cold Spring Harbor, New York.

Sanger, F., S. Nicklen, and A.R. Coulson. 1977. DNA sequencing with chain-terminating inhibitors. Proc. Natl. Acad. Sci. 74: 5463-5467.

Santamaria, P., J. Deatrick, and N.B. Randsholt. 1989. Pattern triplications following genetic ablation on the wing of Drosophila. Effect of eliminating the polyhomeotic gene. Wilhelm Roux's Arch. Dev. Biol. 198: 65-77.

Scott, M.P., A.J. Weiner, B.A. Polisky, T.I. Hazelrigg, V. Pirotta, F. Scalenghe, and T.C. Kaufman. 1983. The molecular organization of the Antennapedia locus of Drosophila. Cell 35: $763-776$.

Simon, J., M. Peifer, W. Bender, and M. O'Connor. 1990. Regulatory elements of the bithorax complex that control expression along the anterior-posterior axis. EMBO /. 9: 39453956.

Smouse, D. and N. Perrimon. 1990. Genetic dissection of a complex neurological mutant, polyhomeotic, in Drosophila. Dev. Biol. 139: 169-185.

Smouse, D., C.S. Goodman, A.P. Mahowald, and N. Perrimon. 1988. Polyhomeotic: A gene required for the embryonic development of axon pathways in the central nervous system of Drosophila. Genes \& Dev. 2: 830-842.

Spierer, P., A. Spierer, W. Bender, and D. Hogness. 1983. Molecular mapping of genetic and chromomeric units in Drosophila melanogaster. J. Mol. Biol. 168: 35-50.

Struhl, G. 1981. A gene product required for correct initiation of segmental determination in Drosophila. Nature 293: 36-41.

Struhl, G. and M. Akam. 1985. Altered distribution of Ultrabithorax transcripts in extra sex combs mutant embryos of Drosophila. EMBO I. 4: 3259-3264.

Vallee, B.L., J.E. Coleman, and D.S. Auld. 1991. Zinc fingers, zinc clusters, and zinc twists in DNA-binding protein do- mains. Proc. Natl. Acad. Sci. 88: 999-1003.

Villares, R. and C.V. Cabrera. 1987. The achaete-scute gene complex of $\mathrm{D}$. melanogaster: Conserved domains in a subset of genes required for neurogenesis and their homology to myc. Cell 50: 415-424.

Wedeen, C., K. Harding, and M. Levine. 1986. Spatial regulation of Antennapedia and bithorax gene expression by the Polycomb locus in Drosophila. Cell 44: 739-748.

Wu, C.-T., R.S. Jones, P.F. Lasko, and W.M. Gelbart. 1989. Homeosis and the interaction of zeste and white in Drosophila. Mol. Gen. Genet. 218: 559-564.

Zink, B. and R. Paro. 1989. In vivo binding pattern of a transregulator of the homeotic genes in Drosophila melanogaster. Nature 337: 468-471.

Zink, B., Y. Engstrom, W.J. Gehring, and R. Paro. 1991. Direct interactions of the Polycomb protein with Antennapedia regulatory sequences in polytene chromosomes of Drosophila melanogaster. EMBO /. 10: 153-162. 


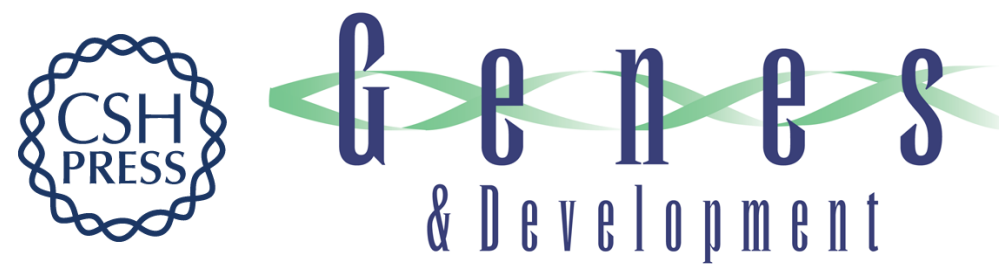

\section{The polyhomeotic gene of Drosophila encodes a chromatin protein that shares polytene chromosome-binding sites with Polycomb.}

M DeCamillis, N S Cheng, D Pierre, et al.

Genes Dev. 1992, 6:

Access the most recent version at doi:10.1101/gad.6.2.223

References This article cites 55 articles, 15 of which can be accessed free at:

http://genesdev.cshlp.org/content/6/2/223.full.html\#ref-list-1

License

Email Alerting

Service

Receive free email alerts when new articles cite this article - sign up in the box at the top right corner of the article or click here.

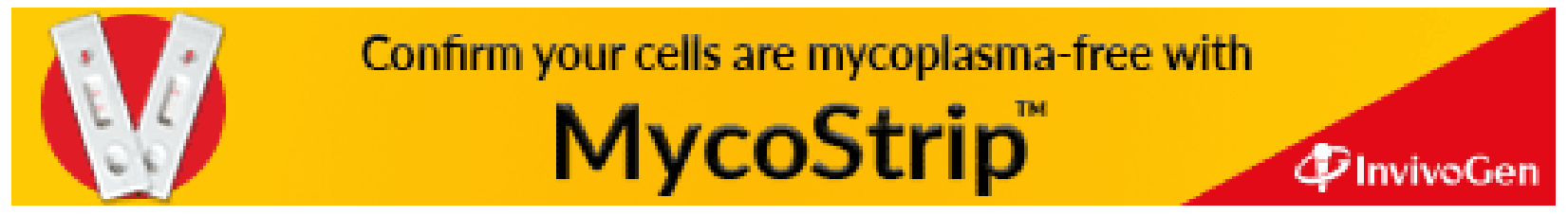

